\title{
On the Anticipatory Precue Activity in Motor Cortex
}

\author{
Joachim Confais, Bjørg Elisabeth Kilavik, Adrián Ponce-Alvarez, and Alexa Riehle \\ Institut de Neurosciences de la Timone, CNRS-Université Aix-Marseille, UMR 7289, 13385 Marseille Cedex 5, France
}

\begin{abstract}
Motor cortical neurons are activated during movement preparation and execution, and in response to task-relevant visual cues. A few studies also report activation before the expected presentation of cues. Here, we study specifically this anticipatory activity preceding visual cues in motor cortical areas. We recorded the activity of 1215 neurons in the motor cortex of two macaque monkeys while they performed a center-out reaching task, including two consecutive delays of equal duration, known in advance. During the first delay (D1), they had to await the spatial cue and only reach to the cued target after the second delay (D2). Forty-two percent of the neurons displayed anticipatory activity during D1. Among these anticipatory neurons, 59\% increased (D1up) their activity and the remaining decreased (D1down) their activity. By classifying the neurons according to these firing rate profiles during D1, we found that the activity during D2 differed in a systematic way. The Dlup neurons were more likely to discharge phasically soon after the spatial cue and were less active during movement execution, whereas the D1down neurons showed the opposite pattern. But, regardless of their temporal activity profiles, the two categories seemed equally involved in early and late motor preparation, as reflected in their directional selectivity. This precue activity in motor cortex may reflect two complementary, coexisting processes: the facilitation of incoming spatial information in parallel with the downregulation of corticospinal excitability to prevent a premature response.
\end{abstract}

\section{Introduction}

Animals, as they anticipate events, use available temporal information to optimize action and perception. This information might, for instance, be extracted from isochronously presented signals, or by observing the motion of a ball in the air. In motor cortical areas, the anticipation (preparation) of a movement has been widely studied (Riehle, 2005), but here we will focus on the activity of the same areas during anticipation of relevant cues.

The primary motor cortex (M1) and dorsal premotor cortex (PMd) are activated during movement execution (Weinrich et al., 1984, 1985; Riehle and Requin, 1989, 1995; Crammond and Kalaska, 1996), but also beforehand, as the animal prepares to move (Wise and Mauritz, 1985; Wise and Kurata, 1989; Shen and Alexander, 1997a,b; Riehle, 2005), and after the presentation of relevant visual cues (Godschalk et al., 1981; Vaadia et al., 1986; Riehle and Requin, 1989; Miller et al., 1992; Boussaoud and Wise, 1993a,b; Crammond and Kalaska, 1996, 2000; Yamagata et al.,

Received April 11, 2012; revised Aug. 28, 2012; accepted Sept. 2, 2012.

Author contributions: B.E.K. and A.R. designed research; J.C., B.E.K., and A.P.-A. performed research; J.C. analyzed data; J.C., B.E.K., and A.R. wrote the paper.

This work was supported by Agence National de la Recherche Grant ANR-NEUR-05-045-1; an AXA Research Fund grant (to B.E.K.); a Consejo Nacional de Ciencia y Tecnología de México (Mexican government) grant (to A.P.-A.); a Ministère de l'enseignement supérieur et de la recherche (French government) grant (to J.C.); and Fondation pour la Recherche Médicale grants (to B.E.K. and J.C.). We thank William A. Mackay and Laurent Goffart for critically reading the manuscript; Emmanuel Procyk and Martine Meunier for making available to us the MRI scanner facility (Institut National de la Santé et de la Recherche Médicale, Lyon, France); Thomas Brochier for generously sharing needed equipment; Ivan Balansard and Marc Martin for animal care; and Joel Baurberg, Alain De Moya, and Xavier Degiovanni for technical assistance. We also thank two anonymous reviewers for insightful comments on an earlier version of this manuscript.

Correspondence should be addressed to Joachim Confais, Institut de Neurosciences de la Timone, Campus Santé Timone, 27 Bd. Jean Moulin, 13385 Marseille Cedex 5, France. E-mail: joachim.confais@univ-amu.fr.

Adrián Ponce Alvarez's present address: Computational Neuroscience Group, Department of Technology, Universitat Pompeu Fabra, Roc Boronat, 138, E-080818 Barcelona, Spain.

DOI:10.1523/JNEUROSCI.1768-12.2012

Copyright $\odot 2012$ the authors $\quad 0270-6474 / 12 / 3215359-10 \$ 15.00 / 0$
2009). Furthermore, Wise and collaborators (Mauritz and Wise, 1986; Vaadia et al., 1988; di Pellegrino and Wise, 1993a) described an anticipatory activity in PMd preceding relevant sensory cues, even when the movement was to be executed after a subsequent delay of up to several seconds. Such anticipatory activity was also reported in other areas, including parietal area 5 (MacKay and Crammond, 1987), the frontal cingulate cortex (Niki and Watanabe, 1979), the dorsal prefrontal cortex (Vaadia et al., 1986; Niki and Watanabe, 1987), and M1 (Crammond and Kalaska, 1996; Fig. 3 in Roux et al., 2003). This widespread phenomenon of preactivation was only observed with predictable cue timing. However, its function is still unclear: it could participate in a general timing process of the cue (Durstewitz, 2004; Coull and Nobre, 2008, Fujioka et al., 2012), or be part of a preparatory process in anticipation of a future motor event, similar to what was found before movement onset (Lucchetti and Bon, 2001; Renoult et al., 2006; Lebedev et al., 2008).

Although very detailed, the studies about precue activity in motor cortex remained descriptive and focused only on neurons with increasing activity, ignoring those with systematic decreasing firing rate preceding the spatial cue (SC). In this study, we addressed both patterns of precue activity (increasing and decreasing), linking them to the subsequent phases of visuomotor transformation and movement preparation. Furthermore, we analyzed neuronal activity of both PMd and M1. We used a task in which the timing of the visual SC was indicated at the start of each trial. Our main finding is that the cue anticipation involves almost half of the neurons of both M1 and PMd, with $60 \%$ of those showing an increasing precue activity and the remaining a decreasing precue activity. The subsequent preparatory activity depends on the direction of the precue activity, especially in the epoch immediately following the cue and during late movement preparation. Despite marked differences in firing rate modula- 
tions, the prevalence of directional selectivity in the two categories of anticipatory neurons is comparable, suggesting a similar degree of involvement in early and late motor preparation. We propose that this anticipatory activity reflects a presetting mechanism to allow an optimal processing of the visual information, while prohibiting a premature response. Preliminary data were presented in Confais et al. (2011).

\section{Materials and Methods}

Animal preparation. Two adult male Rhesus monkeys (T and M, both 9 $\mathrm{kg}$ ) participated in this study. Care and treatment of the animals during all stages of the experiments conformed to the European and French government regulations.

A detailed description of animal preparation and the behavioral task was given in Kilavik et al. (2010) and is briefly outlined here. After learning the task, the monkeys were prepared for multielectrode recordings in the right hemisphere of the motor cortex, contralateral to the trained arm. The chamber locations above motor cortex were verified with T1weighted MRI scans in both monkeys, and intracortical microstimulation (ICMS) allowed further subdivision into M1 and PMd in monkey M (Kilavik et al., 2010). Locations in which microstimulation of $<20 \mu \mathrm{A}$ current induced muscle twitches were defined as M1. Other locations where higher current was needed were denoted PMd. Twitches could also be evoked at the most anterior recording locations, indicating that we remained within the caudal part of PMd. The recording depths $(<2 \mathrm{~mm}$ below the dura) suggested that we recorded from the rostral part of M1 in the precentral gyrus, and not in the anterior bank of the central sulcus. The different recording locations spanned a diameter of $\sim 3$ and $12 \mathrm{~mm}$ on the surface in monkeys $\mathrm{T}$ and $\mathrm{M}$, respectively.

A multielectrode, computer-controlled microdrive (MT-EPS, AlphaOmega) was used to transdurally insert up to four microelectrodes in monkey T and up to eight microelectrodes in monkey M. Signals were amplified and bandpass filtered from 0.3 to $10 \mathrm{kHz}$ to obtain the highfrequency signal, on which an online spike-shape detection method was applied (MSD, AlphaOmega), allowing isolation of up to three spike shapes per electrode. The timing of each spike was then stored as transistor-transistor logic pulses at a temporal resolution of $32 \mathrm{kHz}$. In parallel, the raw, unfiltered signal was digitized and stored at $32 \mathrm{kHz}$, and used to carry out offline spike sorting by Principal Component Analysis-based software [Mclust (Normal Mixture Modeling for Model-Based Clustering, Classification, and Density Estimation), http://www.stat.washington. edu/mclust/] when the online spike sorting was uncertain. Behavioral data were transmitted online to AlphaMap (AlphaOmega) from the Cortex software (NIMH, http://dally.nimh.nih.gov/), which was used to control the task.

Behavioral task. For more than one year, we trained the monkeys to make arm movements from a common center position in six directions in the horizontal plane. However, after the recordings started, in some sessions only two opposite directions were used $(26 \%$ of the sessions in monkey $\mathrm{T}$ and $36 \%$ in Monkey $\mathrm{M})$. In the remainder of the recording sessions, all six directions were used. The monkeys had continuous monitor feedback about hand (cursor) and the six possible target positions (red outlines).

Two delays were presented successively in each trial (Fig. 1A), the first (D1) demanding temporal attention in anticipation of the visual SC and the second (D2) involving visuomotor integration and movement preparation while waiting for the GO signal. Delay duration (short or long) was modulated from trial to trial in a pseudorandom fashion, but was kept the same for both delays within one trial. The monkey started each trial by moving the handle to the center and holding it there for $700 \mathrm{~ms}$ until a temporal cue (TC) was presented. Each TC consisted of a $200 \mathrm{~ms}$ long tone, its pitch indicating the delay duration to be estimated, starting at the end of the tone (low pitch for short delay and high pitch for long delay). For monkey M, one of two visual cues was simultaneously presented centrally on the monitor. Neither of the two animals had visual/auditory-evoked potentials to TC in the local field potential (LFP) (Kilavik et al., 2010) and $<1 \%$ of the recorded neurons responded phasically to TC, suggesting that TC was encoded and used in motor cortex in a similar way in the two monkeys, despite the difference in the sensory modalities of the cue (auditory only vs combined auditory and visual).

For monkey T, the short delay was fixed to $700 \mathrm{~ms}$ and the long delay was fixed to $1500 \mathrm{~ms}$. For monkey M, the short delay was fixed to $1000 \mathrm{~ms}$ and the long delay was fixed to $2000 \mathrm{~ms}$. The delay that followed TC (D1) demanded attention in time (i.e., temporal expectation; Coull and Nobre, 2008) to perceive the SC that was presented very briefly ( $55 \mathrm{~ms}$ ) at the end of the delay at the peripheral target location. To assure the temporal precision of SC illumination, light-emitting diodes (LEDs) were used. These were mounted in front of the computer screen. The SC was subsequently masked by the additional illumination of the five remaining LEDs, marking the start of D2. During D2, the monkey had to remember and prepare for the movement direction indicated by the visual SC. All LEDs went off at the end of D2 (GO signal), indicating to the monkey to perform the movement. Even though the delay durations were slightly different for each monkey, all delays were within the range of 700-2000 $\mathrm{ms}$, where timing performance is rather uniform and expected to depend on the same mechanism (Gibbon et al., 1997; Lewis and Miall, 2009). The two monkeys had similar differences in performance between shortdelay and long-delay trials (see Results). The reaction time (RT) was computed online to reward the monkey according to the outcome of the trial, by detecting when the pointer left the central target (a maximum of $500 \mathrm{~ms}$ RT and $500 \mathrm{~ms}$ movement time to arrive at the peripheral target was allowed). For a better accuracy, the RT used in further analyses was computed again offline, and defined as the moment when the trajectory of at least one of the $x$ and $y$ movement coordinates reached a predefined threshold (Kilavik et al., 2010).

Data analysis. All analyses were conducted using the software Matlab (MathWorks) version 7.10 and 7.12 on a Linux platform.

Averaged activity. The averaged perievent time histogram (PETH) of each neuron category was computed. The mean spike count of each neuron was computed with a temporal resolution of $1 \mathrm{~ms}$ across all movement directions, then smoothed with a Gaussian filter (length, 50 $\mathrm{ms} ; \mathrm{SD}, 15 \mathrm{~ms}$ ) and converted in spikes per second. Then, the smoothed firing rates were averaged separately for different neuron categories (see Fig. 2). To assess the difference of firing rate between different categories of neurons at the population level, we typically used a Mann-Whitney $U$ test on fixed time windows, as described in Results.

Firing rate peak detection. For each neuron, the peak in the mean activity following SC (if any) was detected for each neuron, to compute its latency and amplitude. The direction with the highest spike count in a $200 \mathrm{~ms}$ window following SC (preferred direction) was selected to conduct the analysis independently for each neuron. For each neuron, the PETH was computed with a $1 \mathrm{~ms}$ resolution and smoothed in a $700 \mathrm{~ms}$ time window starting 200 before the onset of the SC. The moment of the peak firing rate (see Fig. $3 B, C$ ) was located using the Matlab function findpeaks, which compares each data point against its two neighbors under the following set of parameters: the peak amplitude (difference between the peak activity and the minimum activity in the window) must be at least 5 spikes/s, and the peak latency $40 \mathrm{~ms}$ or more after SC onset. If several peaks were detected, the one with the highest firing rate was selected. All analyses were subsequently controlled by visual inspection to avoid any spurious peak detection. The same method was used to detect the moment of peak firing rate around movement, except that the data of all trials in one movement direction were aligned to movement onset before computing the smoothed PETH. The direction with the highest firing rate in a $200 \mathrm{~ms}$ epoch centered on movement onset was selected for further analysis. The moment with maximal firing rate was then searched in a $600 \mathrm{~ms}$ window centered on movement onset. As a control, trial-by-trial peak detection was also performed by computing the maximum firing rate in a sliding window in the same epochs, and taking the median latency of this maximum for each neuron. The results were very similar, and only the results based on the method using the averaged activity are reported.

Directional selectivity. The time-resolved directional selectivity of the firing rate during D2 was computed separately for each neuron (see Fig. $5 A$ ). A method based on the vector sum was used to compute the preferred direction of the neuron in a given time bin, as well as the magni- 


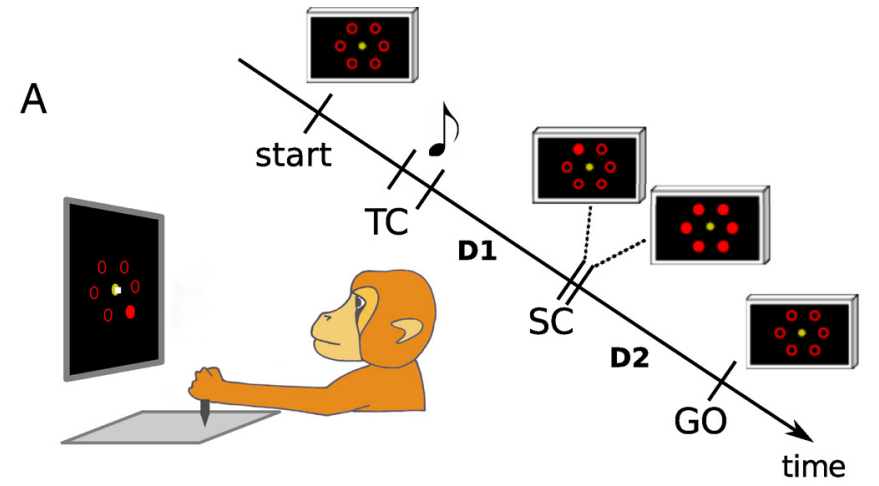

B
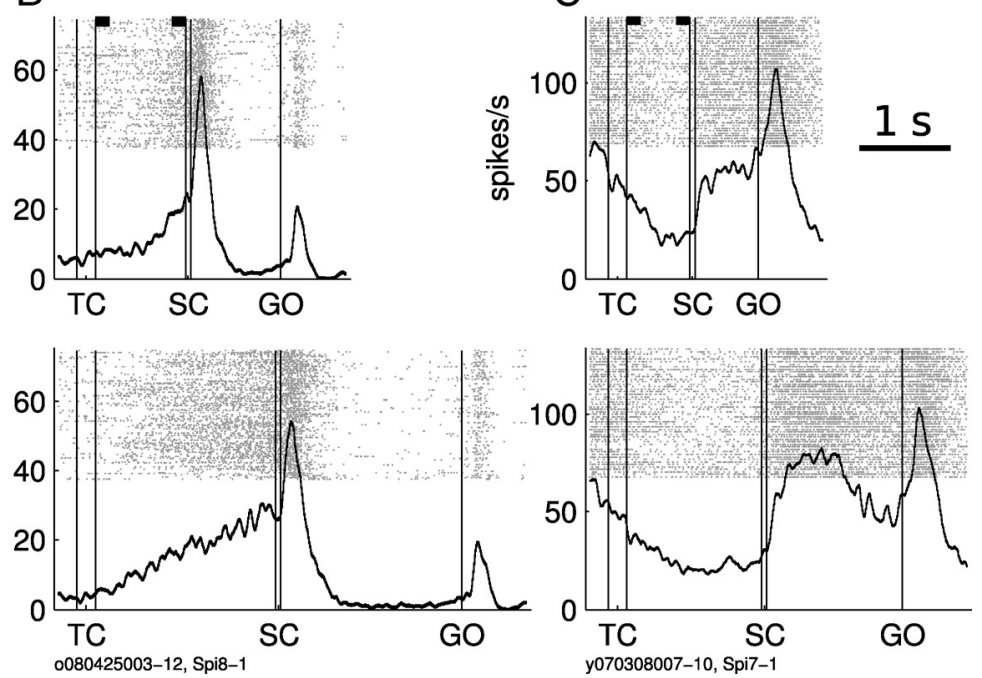

Figure 1. Behavioral paradigm and activity of two representative neurons. $A$, Left, Drawing of the experimental apparatus showing the SC epoch (note the cursor on the central fixation dot). Right, Sequence of task events, not to scale. Start indicates the moment when the monkey brings the cursor to the center of the screen to initiate a new trial. The note indicates the presentation of a tone. Tone pitch differs according to delay duration. All screen-shots shown in the diagram stay on until the next one appears (cursor is not shown). TC, $200 \mathrm{~ms} ; \mathrm{SC}, 55 \mathrm{~ms}$; D1, delay 1, D2, delay 2. Both delays have either short duration (700 ms in monkey T and $1000 \mathrm{~ms}$ in monkey M) or long duration ( $1500 \mathrm{~ms}$ in monkey T and $2000 \mathrm{~ms}$ in monkey M). There is also a $700 \mathrm{~ms}$ delay between start and TC. B, C, Raster plots and PETHs of two neurons (left and right). Top, Short-delay trials; bottom, long-delay trials. All movement directions included. In the raster plots, each dot is an action potential and each row a trial, ordered in chronological order of recording from bottom to top. The thick black lines represent the neuronal activity averaged across all trials and movement directions. The black rectangles on top of each raster display indicate the analysis windows used to classify neurons (see Results).

tude of the directional selectivity. This method has already been applied to LFP data by Kilavik et al. $(2010,2012)$. Only the sessions including six directions were used for this analysis. This rules out the possibility of misclassifying as nonselective neurons that may have a preferred direction orthogonal to the two presented in two-direction sessions. For each neuron in long-delay trials, the mean firing rate in each direction was computed in a sliding window of $200 \mathrm{~ms}$, shifted by $25 \mathrm{~ms}$, from $400 \mathrm{~ms}$ before SC to $900 \mathrm{~ms}$ after GO. Within each time window, the mean firing rate provided the magnitude of six vectors, pointing in the six movement directions. The mean resultant vector was then computed to get the preferred direction and the magnitude of the selectivity (the length of the vector). To test the significance of the tuning, a shuffle test was used: the trials were randomized across the six directions to compute a resultant vector "due to chance." The procedure was repeated 1000 times. If the real vector was longer than $95 \%$ of the shuffle vectors, the tuning was deemed significant. To avoid false positives due to a very low firing rate, a minimum $5 \mathrm{~Hz}$ criterion in the preferred direction of the neuron was added. Additionally, to control for local firing rate fluctuations, only series of at least three significant windows in a row were considered. Figure 5 shows that the amount of directionally selective neurons before the SC is virtually 0 , indicating that our method to control false positives is efficient.
To assess the potential directional tuning of a neuronal population as a whole, a HodgesAjne test (circular statistics toolbox, Berens, 2009) was used: if the test reached significance $(p<0.05)$, the neuronal population deviated from uniformity, and its preferred direction was calculated by a mean resultant vector. Otherwise, the preferred directions of the neurons were too evenly spread, and the population as a whole was not considered tuned. Consequently, the angular difference between the preferred directions of two populations could only be computed if both of them were significantly tuned in the first place. In this case, the angular difference between the mean vectors of the two populations was computed. Finally, to test for angular difference in preferred directions of the two populations in a nonparametric way, the individual vectors in the two populations were shuffled and dispatched at random in two groups, and a new angular distance was computed. The procedure was repeated 1000 times. The angular difference between the two populations was deemed significant if it was larger than $95 \%$ of the random ones.

Comparison of firing rate in short and long trials during D1. To evaluate whether the activity of a neuron has an early selectivity for the duration of the delay, the firing rate in the epoch following TC was compared between short-delay and long-delay trials (see Fig. 6). For each neuron, the spike count during D1 in the short-delay trials was compared with the corresponding epoch in the long-delay trials (see Fig. 2, corresponding to the period between TC and the dashed lines). A sliding window of $100 \mathrm{~ms}$ was shifted in steps of $25 \mathrm{~ms}$, starting $300 \mathrm{~ms}$ before TC and ending at the onset of SC in short-delay trials (and at the same moment in long-delay trials). In each time bin, the spike counts of the short-delay and the long-delay trials were compared (MannWhitney $U$ test) independently for each neuron. Then, the proportion of neurons with a significant difference $(p<0.05)$ was computed for each time bin.

\section{Results}

\section{Neuronal database and behavior}

The behavioral results have already been detailed in Kilavik et al. (2010). Briefly, in both monkeys, RTs were shorter and error rates lower in short-delay trials than in long-delay trials.

In monkey $\mathrm{T}$, the activity of 470 single neurons was recorded in 85 recording sessions. In monkey $\mathrm{M}$, the activity of 745 single neurons was recorded in 147 recording sessions. In monkey $\mathrm{M}$, in which the location of recordings was controlled by ICMS (see Materials and Methods), 251 neurons were assigned to M1 and 489 to PMd. Typically, the activity of each neuron was recorded during 20 correct trials per trial type (direction times delay duration). For monkey $\mathrm{T}$, the activity of 350 neurons was recorded in six-direction sessions, while the activity of 120 neurons was recorded in two-direction sessions. Meanwhile, for monkey M, the activity of 477 neurons was recorded in six-direction sessions, while the activity of 263 neurons was recorded in two-direction sessions. The raster plot of each neuron was inspected visually, and trials with obvious artifacts were removed from analysis (on 
average, $9 \%$ of the trials of $6 \%$ of the neurons in monkey $\mathrm{T}$, and $12 \%$ of the trials of $14 \%$ of the neurons in monkey $\mathrm{M}$ were removed from analysis).

\section{Anticipatory activity during D1}

During D1, between TC and SC, the monkey knew in advance the moment of SC appearance. Thus, the monkey could anticipate the SC. A large number of neurons systematically modulated their firing rate before SC. We labeled such an activity pattern as "anticipatory". Note that here "anticipatory activity" refers to the activity preceding SC, and does not concern the preparatory activity in anticipation of movement execution during D2 (between SC and the GO signal). These anticipatory neurons were defined as D1up neurons if they showed a significant upward modulation of activity, or D1down neurons if they showed significant downward modulation of activity (see below).

Figure $1 B, C$ shows the mean activities and spike trains (raster displays) of two representative neurons from our set with anticipatory activity. The D1up neuron illustrated in Figure $1 B$ has a low firing rate at the beginning of the trial and a systematic increase of activity starting at $\sim 400 \mathrm{~ms}$ after TC in short-delay and long-delay trials. It is worth noting that the onset of the increase in firing rate is variable from trial to trial (see raster displays). As a response to SC, it exhibits a short-latency burst of activity. This pattern of activity is typical, as will be described below. In contrast, the D1down neuron illustrated in Figure $1 C$ shows a continuous decrease in firing rate throughout D1, reaching a minimum just before SC and no burst after SC.

To quantify how many neurons display one of these two prominent activity patterns, a systematic analysis of the firing rate during D1 was conducted. A neuron is defined as anticipatory if its spike count measured in two separate epochs, following TC and preceding SC, (measured in two $150 \mathrm{~ms}$ time windows, marked in black in the raster displays in Fig. $1 B, C$ ) differed significantly within trial (Wilcoxon signed-rank test, $p<0.05$ ). For this analysis, all movement directions of the short-delay trials were pooled, since no spatial information has been provided to the monkey at this moment of the task.

In monkeys $\mathrm{T}$ and $\mathrm{M}$, respectively, 49 and $38 \%$ of the neurons could be classified as anticipatory. Among them, 57 and 60\% were classified as D1 up in monkeys $\mathrm{T}$ and $\mathrm{M}$, respectively, and the remaining neurons as D1down (43 and 40\%, respectively). There were significantly more Dlup than D1down neurons in both animals ( $p<0.01, \chi^{2}$ test). The horizontal bar plots on the right in Figure 2 show these results, but expressed here as a fraction of the total neuronal population: in monkeys $\mathrm{T}$ and $\mathrm{M}$, respectively, 28 and $23 \%$ of the neurons are Dlup, and 21 and $15 \%$ are D1down. The D1flat category contains neurons whose activity is not significantly modulated when comparing the start and end of D1. In monkey $\mathrm{M}$, the same percentages of D1up and D1down neurons were recorded in M1 and PMd (D1up: 25 and 22\%, respectively, $p=0.85, \chi^{2}$ test; D1down: 12 and $\left.16 \%, p=0.55\right)$. All the subsequently described analyses were tested separately in the two areas, but yielded similar results. Therefore, the neurons from M1 and PMd were pooled in all the results.

In summary, almost half of the recorded motor cortical neurons showed an anticipatory activity before the occurrence of a visual cue providing prior information about movement direction. Approximately $60 \%$ of these show an increase of activity, being equally distributed in M1 and PMd.
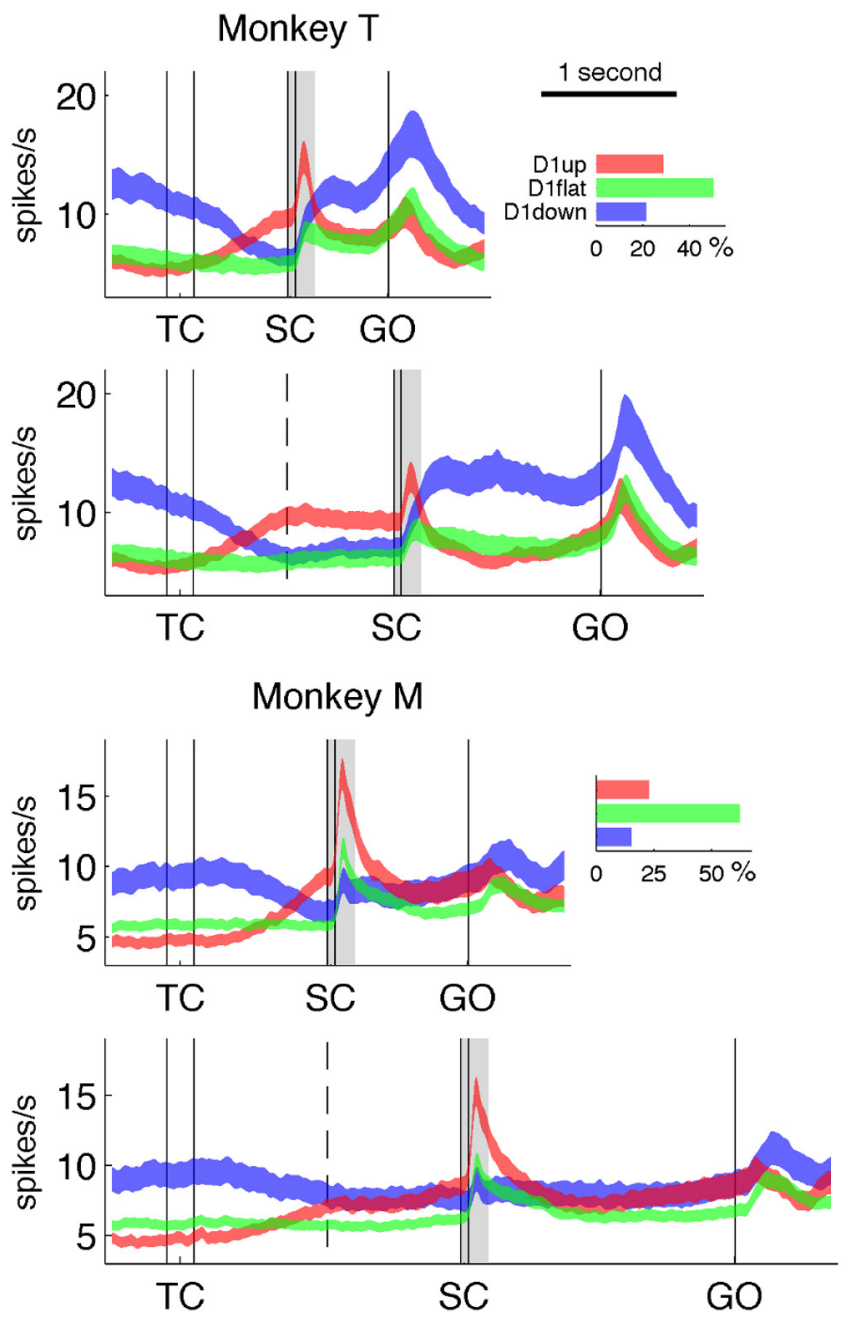

Figure 2. Population activity and proportion of the neurons in the three categories. The curves represent the mean firing rate $\pm S E M$, with the D1up neurons in red, D1down in blue, and D1flat in green. The bar plots on the right represent separately for the two monkeys the percentages of each neuron category among the whole neuronal populations. The dashed lines in the middle of D1 (delay between TC and SC) indicate the time of the SC in short-delay trials. The light gray rectangles illustrate the central analysis window used to classify phasic responses to SC; see Results.

\section{Population activity}

Figure 2 shows the mean firing rate of the three neuron categories, averaged across all movement directions (see Materials and Methods). It is clear that the mean firing rate of the three neuronal categories is modulated differently in time. The D1down neurons have the highest activity before TC, the Dlup neurons show a strong burst of activity shortly after SC, and the D1down neurons seem more active than neurons of the other two categories during movement. The firing rates of the individual neurons in the three categories were measured in three epochs $(200 \mathrm{~ms}$ before TC, $200 \mathrm{~ms}$ after SC, and $200 \mathrm{~ms}$ around movement onset) to examine these differences of activity. The results (Table 1) reveal that (1) the population activity of the D1down neurons is significantly higher than that of the Dlup neurons in the epoch preceding TC ( $p \ll 0.001$ for both monkeys, Mann-Whitney $U$ test across all neurons, with short-delay and long-delay trials pooled); (2) the phasic activity following SC is significantly higher in the Dlup neurons than in the D1down neurons (virtually absent in the latter: $p<0.01$ and $p \ll 0.001$ for monkey T and M, respectively); and (3) the firing rate during movement execution is 
Table 1. Mean firing rates (spikes/s \pm SEM) during different epochs of the trial

\begin{tabular}{lccc}
\hline & Before TC & After SC & Around movement \\
\hline Monkey T & & & \\
$\quad$ D1up $(n=129)$ & $5.6 \pm 0.6$ & $11.9 \pm 1$ & $9.6 \pm 1.1$ \\
D1flat $(n=228)$ & $6.8 \pm 0.8$ & $7.9 \pm 0.8$ & $10.5 \pm 1$ \\
$\quad$ D1down $(n=95)$ & $11.6 \pm 1.1$ & $8.4 \pm 0.9$ & $15.9 \pm 1.8$ \\
Monkey M & & & \\
D1up $(n=168)$ & $4.9 \pm 0.4$ & $13.1 \pm 0.8$ & $9.2 \pm 0.8$ \\
D1flat $(n=455)$ & $6.0 \pm 0.4$ & $8.6 \pm 0.5$ & $8.5 \pm 0.5$ \\
D1down $(n=113)$ & $9.4 \pm 0.9$ & $8.1 \pm 0.8$ & $10.4 \pm 1$ \\
\hline
\end{tabular}

higher in the D1down neurons than in the Dlup neurons, but this is significant only in monkey $\mathrm{T}(p \ll 0.001)$. The mean activity of the D1flat neurons shows less modulation than that of the other neuron categories, which could be due to the possible heterogeneous nature of this category.

If the population is considered as a whole, the mean firing rate of the neurons from the three categories at the end of D1 is not different between short-delay and long-delay trials (MannWhitney $U$ test in a $200 \mathrm{~ms}$ time window, $p=0.85$ and $p=0.71$ in monkey $\mathrm{T}$ and $\mathrm{M}$, respectively). This can be explained by the fact that neurons with a higher firing rate in short-delay trials than in long-delay trials are in the same proportion as neurons showing the opposite pattern. In monkey T, 14\% of neurons are more active in short-delay than in long-delay trials, and $19 \%$ show the opposite pattern ( $\chi^{2}$ test, $\left.p=0.06\right)$. In monkey $\mathrm{M}, 10 \%$ of the neurons are more active in short-delay trials and $12 \%$ are more active in long-delay trials, which is also not significantly different $(p=0.4)$. These results also hold if only the anticipatory neurons are selected ( $p=0.75$ and $p=0.35$ in monkey $\mathrm{T}$ and $\mathrm{M}$, respectively). In summary, the changes of firing rate of all individual neurons seem to balance each other out and lead to the same mean population firing rate before the onset of SC, regardless of the duration of D1.

\section{Characterization of the phasic activity following SC}

By classifying the neurons on the sole basis of their increasing or decreasing firing pattern during D1, two striking differences in their mean activity during D2 emerge (see above). The D1up neurons show a strong burst of activity shortly after the SC, virtually absent in the D1down neurons. Conversely, the D1down neurons are more active than the Dlup neurons during movement execution itself. We therefore studied these two epochs in more detail. The proportion of neurons with an early phasic activity was computed for each neuron category. However, the population peak amplitudes might differ for two reasons: fewer D1down neurons display a phasic activity, and/or their activity peaks have more variable latencies. Two analyses were done to characterize the phasic activity of individual neurons following SC to account for these two possibilities.

First, a neuron was defined as "phasic" if its firing rate was higher in a short epoch following the onset of SC than in the two adjacent periods around it. For each neuron, the single trial spike count of the 200 ms time window shown in gray in Figure 2 was compared with the two adjacent ones (of the same duration) with all movement directions pooled together (two Wilcoxon signedrank tests, $p<0.05$ ). If both tests reached significance, the neuron was classified as "phasic." To temporally dissociate the activity related to SC from the movement-related activity, only long-delay trials were considered. This analysis shows that the probability of having an early phasic activity burst following SC is strongly correlated with the type of activity during D1 (Fig. 3A).
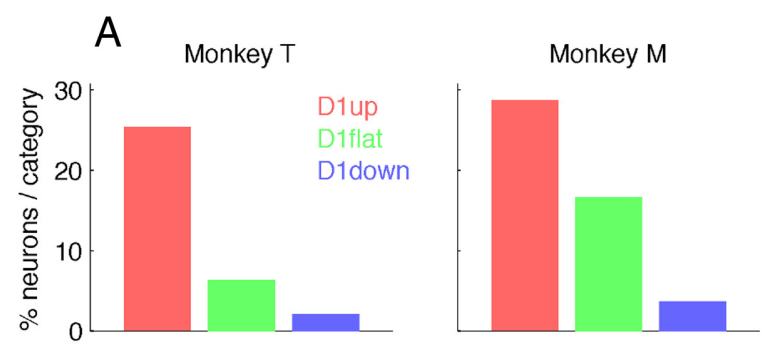

B
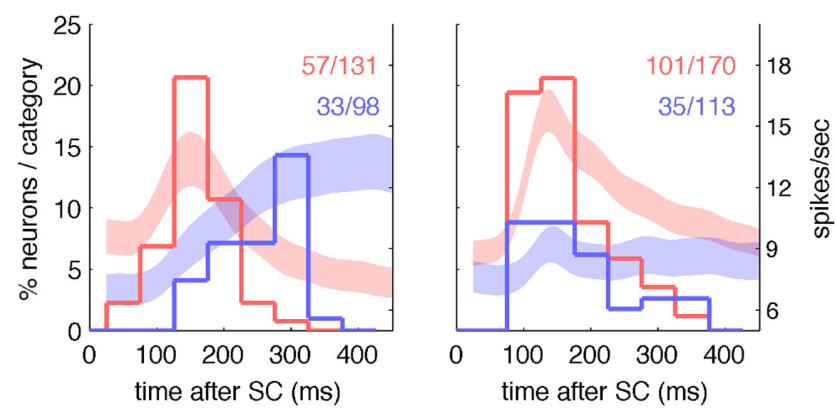

Figure 3. Phasic activity following SC. $A$, Percentages of neurons with a phasic burst of activity in 200 ms following SC, expressed as a fraction of the numbers of neurons per category. The trials of all movement directions of long-delay trials are pooled for this analysis. All comparisons of differences between categories are significant for both monkeys ( $\chi^{2}$ test, $p<$ 0.05), except between D1flat and D1down in monkey T. $\boldsymbol{B}$, Histograms, Distributions of the neurons' peak firing rate latencies, shown as percentages in each category (left ordinate axis). D1up, red; D1down, blue. The digits in the top right corners are the numbers of cells with a detected peak of the total number of neurons in each category. The abscissa represents in milliseconds the peak latency compared with SC onset. For comparison, the PETHs of the two categories in the same epoch have been reproduced in the background, with the firing rate scale indicated on the right ordinate axis. Please note that, unlike the histograms of peak latencies, the PETHs include the activity of all recorded cells, not just the ones with a detected peak.

The percentage of neurons with an early phasic activity burst after $\mathrm{SC}$ is highest in the Dlup neurons, lowest in the D1down neurons, and intermediate in the D1flat neurons. All differences were statistically significant in both monkeys ( $\chi^{2}$ tests, $p<0.05$ ), except between D1flat neurons and D1down neurons in monkey T. Many motor cortical neurons are directionally selective as soon as a stimulus such as SC is presented (Riehle and Requin, 1989; Shen and Alexander, 1997a,b; for review, see Riehle, 2005). If one of the populations of neurons contained more directionally selective neurons than the other, this could artificially increase the firing rate variability when pooling trials across all movement directions. We therefore also tested all neurons using only the preferred direction of each neuron (data not shown). The overall results were the same, but the percentage of significantly phasic neurons decreased slightly (presumably because of the smaller number of included trials).

Second, the latency of the peak of activity after SC was computed for each neuron. The peak was searched in a time window between 40 and $500 \mathrm{~ms}$ after SC [40 ms corresponding to the shortest motor cortical response to a visual stimulation (Riehle, 1991)] only in long-delay trials. The distributions of all peak latencies were then plotted separately for the D1up and D1down neurons in (Fig. $3 B, C$ ). The results from this second analysis confirm those from the first analysis in the sense that more D1up than D1down neurons have an early peak $(<200 \mathrm{~ms})$. However, there are clear differences between the two monkeys. In monkey $\mathrm{T}$, we found similar proportions with a detected peak in both categories of neurons ( 44 and $34 \%$, respectively; $\chi^{2}$ test, $p=$ 
$0.15)$, but the median peak latencies were significantly shorter in the Dlup than in the D1down neurons (151 and $250 \mathrm{~ms}$, respectively; Mann-Whitney $U$ test, $p \ll 0.001)$. In monkey $\mathrm{M}$, the median peak latencies were not different between Dlup and D1down neurons (145 and $162 \mathrm{~ms}, p=0.22$ ), but significantly more D1up than D1down neurons showed a peak of activity (59 and $31 \%, p \ll 0.01)$. These differences in peak latency distributions are clearly reflected in the mean population activity shown in Figure 2, which is reproduced in the background of Figure 3. In monkey $\mathrm{T}$, the response to SC of the D1down neurons seems delayed compared with the D1up activity, but has a similar amplitude, whereas in monkey $\mathrm{M}$, all categories of neurons show a short-latency change of activity, but with a higher amplitude and a more phasic response in the Dlup than in the D1down neurons. In conclusion, the second analysis confirms that the D1up neurons are more likely to have a burst of activity in the early epoch following SC than the D1down neurons.

Using the same method as in the previous paragraph, but in a $600 \mathrm{~ms}$ time window centered on movement onset, the same analysis was performed on the movement-related activity. The distributions of the motor peak latencies of the D1up and D1down neurons are largely overlapping, but significantly different in monkey T $(p<0.01)$, with Dlup neurons peaking on average $43 \mathrm{~ms}$ earlier than D1down neurons. The same tendency can be seen in monkey $M(p=0.06)$, with a mean difference of $36 \mathrm{~ms}$.

\section{Comparison of visual-related and movement-related activity}

As described above, the Dlup neurons discharge, on average, with a higher firing rate during the epoch following SC than during movement execution, whereas the D1down neurons exhibit the opposite firing pattern (Fig. 2). However, this difference in mean activity may be due to a high firing rate of a small number of dominant neurons. To rule out this possibility, the discharge evoked by SC and that related to movement execution were compared for each neuron (Fig. 4). The single-trial spike counts in two $200 \mathrm{~ms}$ time windows were compared: the first one following $\mathrm{SC}$ and the second around movement onset. Figure 6 shows for each monkey the proportion of D1up, D1flat, and D1down neurons with a significant (Wilcoxon signed-rank test, $p<0.05$ ) higher spike count in the visual than in the motor epoch (left), and with a significant higher spike count in the motor than in the visual epoch (right). The proportion of neurons with a higher firing rate during the visual than during the motor epoch is highest in the D1up neurons and lowest in the D1down neurons. Conversely, the D1down category has the highest proportion of neurons with a preference for the motor over the visual epoch, followed by the D1flat and then the D1up categories. Note that the proportion of neurons with a higher firing rate in the visual epoch cannot be accounted for solely by the presence of an early phasic activity. For example, this phasic activity is only present in $30 \%$ of the Dlup neurons of monkey M (Fig. 3A), and Figure 4 shows that twice as many Dlup neurons are more active in the visual than in the motor epoch. In summary, neurons that increase their activity before the SC have a tendency to exhibit a short-latency burst following the cue, and to have in general a higher firing rate during the first part of D2 than during movement execution. In contrast, neurons that decrease their firing rate before the SC tend to be quieter in the early epoch following it, and are more active during movement execution than during the early processing of the visual cue. This may suggest that the two populations of neurons are sequentially involved in movement preparation.
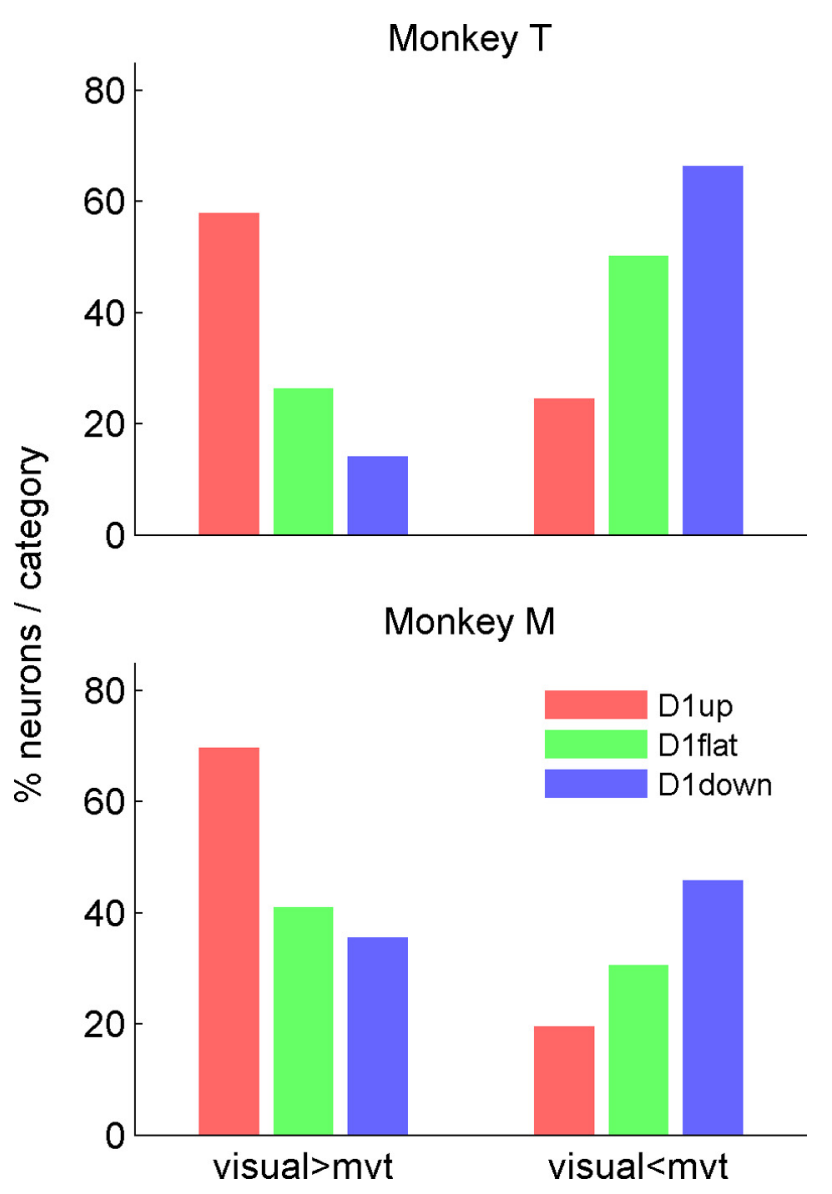

Figure 4. Differences in firing rates between the visual and motor epochs. Proportions of neurons in each category with a significantly higher activity in the "visual" than in the "motor" epoch (left), and with a significant higher activity in the "motor" than in the "visual" epoch (right). Only long-delay trials are used, and all movement directions are considered. Because only the neurons with significant differences are shown here, the total percentage within each neuron category does not necessarily add up to 100. All comparisons of differences between categories are significant for both monkeys ( $\chi^{2}$ test, $p<0.05$ ), except the difference between D1flat and D1down for visual $>$ mvt in monkey M. mvt, movement.

\section{Directional selectivity}

If neurons are involved in the preparation of a motor act, their activity should reflect some movement parameters, such as its direction. In other words, if the D1up and D1down neurons are involved in different phases of movement preparation, one might expect the activity to be differentially selective to movement direction, depending on task epoch. The time-resolved ratio of neurons being selective for movement direction was therefore computed separately for the three categories, to evaluate whether the evolution of this ratio differed across categories. We assessed the directionality of the neurons with a method based on the vector sum in a $200 \mathrm{~ms}$ sliding window shifted by $25 \mathrm{~ms}$ (see Materials and Methods). The results are shown in Figure $5 \mathrm{~A}$ for long-delay trials. Only the sessions with six target directions were included. First, two epochs of increased directional selectivity emerge: the epochs following SC and around movement onset. In addition, all three neuron categories have significant $(p<0.05)$ proportions of tuned neurons. Second, the two categories of anticipatory neurons (D1up and D1down) generally contain higher proportions of significantly directionally selective neurons than the D1flat category. This might be due to the fact that the D1flat neurons did not have to pass any statistical test to be classified as such, and therefore could include noisier or less task-related 


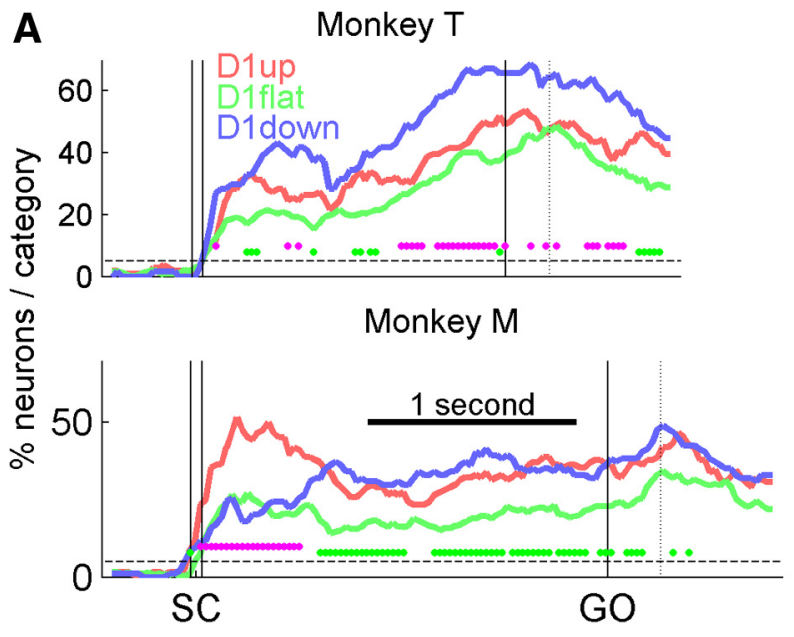

B

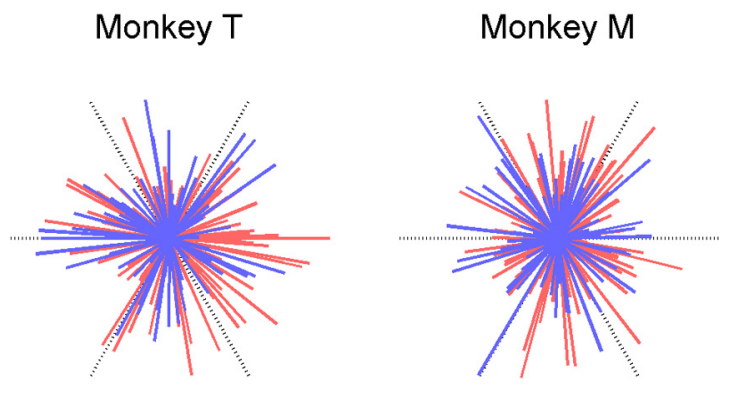

Figure 5. Directional selectivity. $\boldsymbol{A}$, Percentages of neurons (fractions of the sample size of each neuron category) with significant directional selectivity, calculated in a $200 \mathrm{~ms}$ sliding window, shifted by $25 \mathrm{~ms}$. Each magenta dot indicates a window with a significant difference between the D1up and D1down categories $\left(\chi^{2}\right.$ test, $p<0.05$ ). Each green dot indicates a significant difference between the D1flat category and the lowest of the D1up and D1down categories. The dashed lines indicate the $5 \%$ proportion expected by chance. The dotted vertical lines indicate mean RTs. Only neurons recorded in sessions with six directions are used. $\boldsymbol{B}$, Distributions of the directional vectors of the D1up neurons (red) and D1down neurons (blue), selected in the bin of maximum (significant) magnitude for each neuron. Each vector indicates the preferred direction of a neuron and its magnitude of selectivity. The black dashed lines indicate the directions of the six targets, and their length indicates the unit vector (maximum vector length). All distributions are homogeneous. All neurons that were significantly tuned at least once during D2 are considered: 92 of 106 for D1up and 65 of 67 for D1down in monkey T, and 109 of 124 and 72 of 94 in monkey M.

neurons. Third, and most important here, the overall levels of directional selectivity of the two categories of anticipatory neurons have comparable magnitudes, albeit with more D1down neurons tuned than D1up neurons in monkey T and more D1up neurons tuned after SC in monkey $\mathrm{M}$. Indeed, no systematic differences of directional selectivity between the D1up and D1down populations can be seen for the two monkeys related to the different task epochs. The overall differences in tuning observed between monkeys could be due to the localization of the electrodes: we mainly recorded in PMd in monkey M, but we do not have this information for monkey $\mathrm{T}$. In summary, the sequentiality observed in the firing rate patterns of the two categories of neurons (Figs. 2-4) is not reflected in their directional selectivity.

In the work of Mauritz and Wise (1986), the anticipatory activity they observed was interpreted as the anticipation of a rightward movement instruction. In other words, the anticipatory activity would merely be a reflection of the preparation of a default movement direction. Even though there is a major difference between their paradigm and ours (they used only 2 directions, and we mainly used 6 directions), their interpretation could also apply to our data. In our case, the mean RTs of the monkeys in the different movement directions seem to indicate that the behavioral preferred direction (the one with the shortest mean RTs) remained stable throughout the recordings. If we assume that this would also be the case for the putative default direction that the animals would be expecting during D1, it allows us to directly test the interpretation proposed by Mauritz and Wise (1986). According to this hypothesis, neurons with a preferred direction close to the preferred behavioral direction would be the D1up, and the ones with a preferred direction close to the opposite direction would be the D1down. Thus, as populations, the distributions of the preferred directions of the Dlup and D1down neurons should then be unimodal, and have close to opposite selectivity during D2.

Aiming to test this, we first compared the distributions of the preferred directions of the significant directionally selective Dlup and D1down neurons in the two task-relevant epochs mentioned above (Fig. 5A), $200 \mathrm{~ms}$ following SC and around movement onset (trials aligned to movement onset). In none of these two epochs were both populations tuned (data not shown). However, it is possible that the neurons are tuned at different moments during D2. Thus, restricting the measure of directional selectivity to these two epochs may not give an accurate picture of the directional selectivity of the two neuronal populations. To compensate for this possible bias, we allowed all neurons to have their own "moment in time" of maximal directional selectivity: for each neuron the preferred direction was selected in the epoch with its strongest directional selectivity (the epoch with the longest significant mean vector). The distributions of these preferred directions are shown in Figure $5 B$ : all distributions are homogeneous (Hodges-Ajne test, $p=0.5$ and 0.95 for D1 up and D1down in monkey $\mathrm{T}$, and $p=0.72$ and 0.73 in monkey $\mathrm{M}$, respectively). These results suggest that either by taking meaningful epochs of the task, or by pooling together all the possible selective epochs during D2, no striking difference in preferred directions can be detected between D1up and D1down neurons. It is therefore unlikely that the anticipatory activity is explained by early directional selectivity.

\section{Comparison of correct and error trials}

We postulated that the anticipatory activity in D1 could influence the detection and processing of SC. Monkeys T and M made 21 and $15 \%$ of directional errors, respectively (i.e., reaching to the wrong target), indicating that although they were overtrained, the task was still difficult. These directional errors could be (in part) due to the inability of the monkey to correctly identify the $\mathrm{SC}$ in some trials. If the activity of the anticipatory neurons in D1 influences the probability of detecting SC, it should be different when the monkey selected the correct target than when he made an error. The firing rate of the $200 \mathrm{~ms}$ preceding SC of correct and error trials was compared in two different ways. First, the spike count in the window was compared across single trials for each neuron (Mann-Whitney $U$ test), and the percentage of significant neurons calculated for the three categories. Second, the activity in all correct and error trials was averaged separately for each neuron, and a Wilcoxon signed-rank test was computed across all neurons for each of the three categories. Both analyses yielded the same results: there was no significant difference in activity preceding the SC between correct and error trials in any of the neuron categories. 


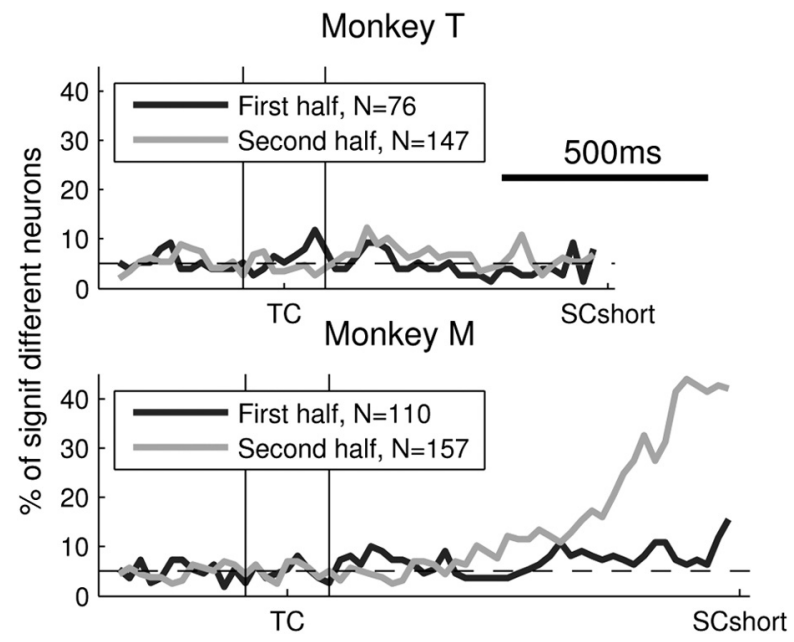

Figure 6. Selectivity to delay duration during D1. Proportions of anticipatory neurons with an early selectivity to delay duration during D1, according to the period of recording. SCshort, onset of the SC in short-delay trials, and equivalent timing in long-delay trials.

\section{Behavioral strategies}

Figure 2 shows that the mean activity during D1 in long-delay trials is different between the two monkeys. This difference could be due to the variety of strategies that the monkey may have used to solve the task. One strategy may be to use the temporal information contained in TC to expect SC at the correct moment. The second strategy would be to expect SC at the end of the short delay in all trials. If then it does not appear, the monkey would know that it is a long-delay trial. In fact, we noted that in monkey $\mathrm{M}$, the firing rate profile during D1 changed along the recording sessions. To quantify these differences, we compared the firing rate during D1 in short-delay trials and the corresponding first half of the long-delay trials. This was done separately for the first and second halves of the recording sessions (that is, for two periods of 3 months in monkey T, and of 5 months in monkey M). Figure 6 shows the proportion of anticipatory neurons with a significantly different firing rate in short-delay and long-delay trials (MannWhitney $U$ test, $p<0.05$, see Materials and Methods) separately for each recording period. In monkey $\mathrm{T}$, the proportion of neurons with a different firing rate in short-delay and long-delay trials never deviates from chance level (dashed line) in the first or in the last recording sessions. Monkey $\mathrm{M}$ shows the same pattern as monkey $\mathrm{T}$ in the first recordings, but a completely different one in the second half of the recording sessions. In the late sessions, the activity of up to $40 \%$ of the anticipatory neurons differed in short-delay and long-delay trials already shortly after TC, as a function of the temporal information provided by TC. These results suggest that monkey $\mathrm{M}$ initially adopted the same strategy as monkey $\mathrm{T}$ (always expecting the SC at the end of the short delay), but then switched to another strategy during later sessions (using the temporal information of TC). Nevertheless, it seems that this possible change of strategy in monkey $\mathrm{M}$ did not have a striking influence on behavior, since the error rate does not change between the two halves of the recording sessions $\left(\chi^{2}\right.$ test, $p=0.75)$. Additionally, even though the mean reaction time of monkey $\mathrm{M}$ decreased by $21 \%$ between early and late sessions, monkey $\mathrm{T}$ showed a decrease of $43 \%$, without displaying the change of activity during D1. To see whether the possible change in behavioral strategy by monkey $\mathrm{M}$ influenced the neuronal activity patterns in D2, all analyses presented above were repeated in monkey $\mathrm{M}$ by separating the data according to the date of recording (first vs second half of the sessions). No qualitative difference was observed. In particular, we observed no significant change in the proportion of anticipatory neurons, or in the phasic response to $\mathrm{SC}$, or in the temporal profile of the directional selectivity. In conclusion, these results suggest that the possible switch of strategy adopted by monkey $\mathrm{M}$ did not change the observed neuronal activity in second part of the trial.

\section{Discussion}

We here demonstrate that almost half of the neurons in M1 and PMd exhibit anticipatory activity preceding the expected presentation of a relevant $\mathrm{SC}$, well before movement parameters can be processed. Of those, $60 \%$ increase their firing rate, whereas the remaining neurons decrease their activity. This anticipatory activity during the first, nonmotor delay is predictive for the temporal profile of the activity during the second, preparatory delay. The difference in activity between neuron categories is especially prominent during the early epoch following the cue, where the probability of having a phasic burst of activity depends upon the type of anticipatory activity. However, both types of anticipatory neurons seem to be similarly involved in motor preparation, as reflected by comparable proportions of directionally selective neurons.

\section{Anticipation and processing of task-related information}

Three studies by Wise and collaborators (Mauritz and Wise, 1986; Vaadia et al., 1988; di Pellegrino and Wise, 1993a) addressed anticipatory activity as defined here (i.e., neuronal activity changes in motor cortex occurring before the presentation of task-relevant information). Our results confirm theirs, even if they mainly focused on PMd and on neurons that we call D1up: (1) the proportion of anticipatory neurons is close to ours (between 30 and $50 \%$ of the neuronal population) and (2) Mauritz and Wise (1986) show that most of their Dlup neurons (46 of 52) exhibit phasic activity following the SC. Thus, it seems that the anticipatory activity we describe is not simply related to our specific task, but a pattern of activity consistently present in motor cortical areas when the monkey can predict the timing of relevant spatial information. Our study is the first one to thoroughly study the D1down neurons, showing that their modulation of activity during the processing of spatial information and during movement preparation clearly differs from that of the Dlup neurons. Furthermore, even though the activity profiles of Dlup and D1down neurons are different, their involvement in the task seems similar, as reflected in the proportions of directionally selective neurons. Finally, this anticipatory activity seems similar in M1 and PMd, even though this could be explained by the fact that we recorded rostrally in M1 and caudally in PMd. MacKay and Crammond (1987) reported an anticipatory activity preceding visual stimuli in parietal area 5 of the monkey. The posterior part of this area projects directly to PMd, and the anterior part to M1 (Geyer et al., 2000). We interpreted this activity in terms of predictions of upcoming events based on experience. Our data suggest that these predictions might then be fed to motor cortex to facilitate the future processing of the information provided by an instruction signal.

Several studies have shown that the information about movement parameters (such as direction) is processed as soon as it is presented ("preprocessing," Requin, 1985; Riehle and Requin, 1989; for review, see Riehle, 2005). This early processing could be reflected in motor areas by the so-called "signal-related" activity (Weinrich and Wise, 1982; Riehle and Requin, 1989; Crammond and Kalaska, 2000; Cisek and Kalaska, 2005), a short-latency 
$(<200 \mathrm{~ms})$ phasic activity following the SC. We find that the proportion of neurons with a peak latency of $<200 \mathrm{~ms}$ following SC drops dramatically from the D1up to the D1down category (Fig. $3 B, C$ ). This suggests that the signal-related neurons are much more likely to display an increasing activity preceding the SC than the others. Furthermore, signal-related activity (Wise et al., 1992; Boussaoud and Wise, 1993a,b; di Pellegrino and Wise, 1993a,b; Crammond and Kalaska, 2000) and precue anticipatory activity (Vaadia et al., 1988; di Pellegrino et Wise, 1993a) are both modulated by the motor meaning of the SC (i.e., whether or not the $\mathrm{SC}$ is informative regarding the movement to be executed), suggesting a functional link between the two patterns of activity. Altogether, this supports the idea that when the timing of an SC is known in advance, motor cortex will enter into a preparatory state, enabling an optimal response.

\section{Interpretations of the anticipatory activity}

Our task was attentionally very demanding, as it required waiting for the presentation of the very brief SC at a precise moment. Rolke and Hofmann (2007) showed that temporal uncertainty, which increases with delay duration (Gibbon, 1977), degrades perceptual processing. This could in part explain the higher rate of directional errors in long than in short trials (even though other causes could intervene, like the monkey being more distracted during the longer D2). The activity seen during D1 could therefore reflect attentional processes, as it has been shown in PMd in other tasks (Boussaoud, 2001; Lebedev and Wise, 2001). Yet, in the trials where the monkey reached to a wrong target, the firing rate preceding SC was not different from that in the correct trials. Thus, even though the anticipatory activity of the motor areas during D1 relies on temporal attention, we could not demonstrate that it influences directly the ability to perceive SC or to choose the correct target.

It was proposed that motor cortical areas could participate in a timing network, when a motor action is to be planned. Lebedev et al. (2008) decoded the duration before or after the initiation of a self-triggered movement from the activity of PMd neurons, and Lucchetti and Bon (2001) showed that the discharge onset of motor cortical neurons depended on the delay before GO. These neurons had ramping activity (increasing or decreasing) similar to what we found in D1. Such a ramping activity has been shown to be a possible substrate of timing processes (Durstewitz, 2004). fMRI studies in humans on implicit timing (i.e., duration does not have to be overtly reported) show frequent activation of the premotor cortex (Coull and Nobre, 1998; Coull et al., 2000), even when no motor act is involved (Schubotz and von Cramon, 2001). Fujioka et al. (2012) showed that in absence of movement, the synchronization of beta oscillations of the MEG signal in the motor cortex were timed to an isochronous signal. This supports the idea that PMd is part of a temporal prediction network (Coull and Nobre, 2008). Recently, Davranche et al. (2011) showed that the BOLD signal of the motor areas (M1 and PM) and of the left intraparietal sulcus (IPS) covaried during a temporal orienting task, but only when the subject had to respond with a movement. This context-dependent recruitment may indicate the modulation of a motor anticipatory process in the motor cortex by temporal information being processed in IPS. Applied to our task, it would mean that the anticipatory activity seen during D1 could reflect the involvement of the motor system in the estimation of the duration preceding the cue. Furthermore, our data suggest that this timing process could set the motor cortex in a particular (optimal) state before the onset of the cue. We find that, at the time of the cue onset, the same population firing rate is reached despite the fact that $22-32 \%$ of the neurons change their firing rate according to delay duration. Saleh et al. (2010) showed that the power of LFP beta oscillations in the motor cortex of a human subject increased before the onset of an attended relevant cue. We speculate that this precue optimal state reflects two complementary processes: one (represented by Dlup neurons) would tune up the gain of cue-processing networks, while the other (D1down neurons) would transiently inhibit the activity of the pyramidal movement-related neurons, to prevent any spurious movement triggering. This might also explain why D1down neurons are directionally selective just after SC. Thus, we can make a prediction about activity during the early epoch following SC. The instantaneous population vectors (Georgopoulos et al., 1983) of the D1up and D1down neuronal populations would point in different directions from trial to trial (according to SC), but would systematically stay opposite to each other. The former would represent the planned movement, while the latter would cancel its immediate execution (Riehle et al., 2006). To confirm this prediction, one would need to analyze data from simultaneous recordings of large populations of anticipatory neurons, which is not the case for our dataset.

\section{Conclusion}

In this study, we showed that many motor cortical neurons display an anticipatory activity preceding the onset of a relevant cue. Their activity during the motor preparatory part of the task is modulated according to their precue activity, especially during the early epoch following the onset of the relevant cue and during movement execution. We propose that whenever there is temporal information available allowing cue anticipation, motor cortex, presumably along with other areas involved in time estimation or motor preparation, enters into an optimal preparatory state, enabling a more efficient processing of the incoming spatial information or its transformation into a motor command ("presetting"; Requin, 1985), while preventing a premature response in tasks that require delayed motor response.

\section{References}

Berens P (2009) CircStat: a Matlab toolbox for circular statistics. J Stat Soft 31. Available at http://www.jstatsoft.org/v31/i10.

Boussaoud D (2001) Attention versus intention in the primate premotor cortex. Neuroimage 14:S40-S45.

Boussaoud D, Wise SP (1993a) Primate frontal cortex: neuronal activity following attentional versus intentional cues. Exp Brain Res 95:15-27.

Boussaoud D, Wise SP (1993b) Primate frontal cortex: effects of stimulus and movement. Exp Brain Res 95:28-40.

Cisek P, Kalaska JF (2005) Neural correlates of reaching decisions in dorsal premotor cortex: specification of multiple direction choices and final selection of action. Neuron 45:801-814.

Confais J, Kilavik B, Ponce-Alvarez A, Riehle A (2011) Visually-related anticipatory activity in motor cortical neurons predicts their involvement in preparatory processes. Program No. 803.3. 2011 Neuroscience Meeting Planner. Washington, DC: Society for Neuroscience.

Coull JT, Nobre AC (1998) Where and when to pay attention: the neural systems for directing attention to spatial locations and to time intervals as revealed by both PET and fMRI. J Neurosci 18:7426-7435.

Coull J, Nobre A (2008) Dissociating explicit timing from temporal expectation with fMRI. Curr Opin Neurobiol 18:137-144.

Coull JT, Frith CD, Büchel C, Nobre AC (2000) Orienting attention in time: behavioural and neuroanatomical distinction between exogenous and endogenous shifts. Neuropsychologia 38:808-819.

Crammond DJ, Kalaska JF (1996) Differential relation of discharge in primary motor cortex and premotor cortex to movements versus actively maintained postures during a reaching task. Exp Brain Res 108:45-61.

Crammond DJ, Kalaska JF (2000) Prior information in motor and premotor cortex: activity during the delay period and effect on pre-movement activity. J Neurophysiol 84:986-1005. 
Davranche K, Nazarian B, Vidal F, Coull J (2011) Orienting attention in time activates left intraparietal sulcus for both perceptual and motor task goals. J Cogn Neurosci 23:3318-3330.

di Pellegrino G, Wise SP (1993a) Visuospatial versus visuomotor activity in the premotor and prefrontal cortex of a primate. J Neurosci 13:1227-1243.

di Pellegrino G, Wise SP (1993b) Effects of attention on visuomotor activity in the premotor and prefrontal cortex of a primate. Somatosens Mot Res $10: 245-262$.

Durstewitz D (2004) Neural representation of interval time. Neuroreport 15:745-749.

Fujioka T, Trainor LJ, Large EW, Ross B (2012) Internalized timing of isochronous sounds is represented in neuromagnetic beta oscillations. J Neurosci 32:1791-1802.

Georgopoulos AP, Caminiti R, Kalaska JF, Massey JT (1983) Spatial coding of movement: a hypothesis concerning the coding of movement direction by motor cortical populations. Exp Brain Res [Suppl] 7:327-336.

Geyer S, Matelli M, Luppino G, Zilles K (2000) Functional neuroanatomy of the primate isocortical motor system. Anat Embryol 202:443-474.

Gibbon J (1977) Scalar expectancy theory and Weber's law in animal timing. Psychol Rev 84:279-325.

Gibbon J, Malapani C, Dale CL, Gallistel C (1997) Toward a neurobiology of temporal cognition: advances and challenges. Curr Opin Neurobiol $7: 170-184$.

Godschalk M, Lemon RN, Nijs HG, Kuypers HG (1981) Behaviour of neurons in monkey peri-arcuate and precentral cortex before and during visually guided arm and hand movements. Exp Brain Res 44:113-116.

Kilavik BE, Confais J, Ponce-Alvarez A, Diesmann M, Riehle A (2010) Evoked potentials in motor cortical local field potentials reflect task timing and behavioral performance. J Neurophysiol 104:2338-2351.

Kilavik BE, Ponce-Alvarez A, Trachel R, Confais J, Takerkart S, Riehle A (2012) Context-related frequency modulations of macaque motor cortical LFP beta oscillations. Cereb Cortex 22:2148-2159.

Lebedev MA, Wise SP (2001) Tuning for the orientation of spatial attention in dorsal premotor cortex. Eur J Neurosci 13:1002-1008.

Lebedev MA, O'Doherty JE, Nicolelis MA (2008) Decoding of temporal intervals from cortical ensemble activity. J Neurophysiol 99:166-186.

Lewis PA, Miall RC (2009) The precision of temporal judgement: milliseconds, many minutes, and beyond. Philos Trans R Soc Lond B Biol Sci 364:1897-1905.

Lucchetti C, Bon L (2001) Time-modulated neuronal activity in the premotor cortex of macaque monkeys. Exp Brain Res 141:254-260.

MacKay WA, Crammond DJ (1987) Neuronal correlates in posterior parietal lobe of the expectation of events. Behav Brain Res 24:167-179.

Mauritz KH, Wise SP (1986) Premotor cortex of the rhesus monkey: neuronal activity in anticipation of predictable environmental events. Exp Brain Res 61:229-244.

Miller J, Riehle A, Requin J (1992) Effects of preliminary perceptual output on neuronal activity of the primary motor cortex. J Exp Psychol Hum Percept Perform 18:1121-1138.

Niki H, Watanabe M (1979) Prefrontal and cingulate unit activity during timing behavior in the monkey. Brain Res 171:213-224.

Renoult L, Roux S, Riehle A (2006) Time is a rubberband: neuronal activity in monkey motor cortex in relation to time estimation. Eur J Neurosci 23:3098-3108.

Requin J (1985) Looking forward to moving soon. Ante factum selective processes in motor control. In: Attention and performance XI (Posner M, Marin O, eds), pp 147-167. Hillsdale, NJ: Lawrence Erlbaum.

Riehle A (1991) Visually induced signal-locked neuronal activity changes in precentral motor areas of the monkey: hierarchical progression of signal processing. Brain Res 540:131-137.

Riehle A (2005) Preparation for action: one of the key functions of the motor cortex. In: Motor cortex in voluntary movements: a distributed system for distributed functions (Riehle A, Vaadia E, eds), 213-240. Boca Raton, FL: CRC.

Riehle A, Requin J (1989) Monkey primary motor and premotor cortex: single-cell activity related to prior information about direction and extent of an intended movement. J Neurophysiol 61:534-549.

Riehle A, Grammont F, MacKay WA (2006) Cancellation of a planned movement in monkey motor cortex. Neuroreport 17:281-285.

Rolke B, Hofmann P (2007) Temporal uncertainty degrades perceptual processing. Psychonomic Bull Rev 14:522-526.

Saleh M, Reimer J, Penn R, Ojakangas CL, Hatsopoulos NG (2010) Fast and slow oscillations in human primary motor cortex predict oncoming behaviorally relevant cues. Neuron 65:461-471.

Roux S, Coulmance M, Riehle A (2003) Context-related representation of timing processes in monkey motor cortex. Eur J Neurosci 18:1011-1016.

Schubotz RI, von Cramon DY (2001) Functional organization of the lateral premotor cortex: fMRI reveals different regions activated by anticipation of object properties, location and speed. Brain Res Cogn Brain Res 11:97-112.

Shen L, Alexander GE (1997a) Neural correlates of a spatial sensory-tomotor transformation in primary motor cortex. J Neurophysiol 77:11711194.

Shen L, Alexander GE (1997b) Preferential representation of instructed target location versus limb trajectory in dorsal premotor area. J Neurophysiol 77:1195-1212.

Vaadia E, Benson DA, Hienz RD, Goldstein MH Jr (1986) Unit study of monkey frontal cortex: active localization of auditory and of visual stimuli. J Neurophysiol 56:934-952.

Vaadia E, Kurata K, Wise SP (1988) Neuronal activity preceding directional and nondirectional cues in the premotor cortex of rhesus monkeys. Somatosens Motor Res 6:207-230.

Weinrich M, Wise SP (1982) The premotor cortex of the monkey. J Neurosci 2:1329-1345.

Weinrich M, Wise SP, Mauritz KH (1984) A neurophysiological study of the premotor cortex in the rhesus monkey. Brain 107:385-414.

Wise SP, Kurata K (1989) Set-related activity in the premotor cortex of rhesus monkeys: effect of triggering cues and relatively long delay intervals. Somatosens Motor Res 6:455-476.

Wise SP, Mauritz KH (1985) Set-related neuronal activity in the premotor cortex of rhesus monkeys: effects of changes in motor set. Proc R Soc Lond B Biol Sci 223:331-354.

Wise SP, Di Pellegrino G, Boussaoud D (1992) Primate premotor cortex: dissociation of visuomotor from sensory signals. J Neurophysiol 68:969-972.

Yamagata T, Nakayama Y, Tanji J, Hoshi E (2009) Processing of visual signals for direct specification of motor targets and for conceptual representation of action targets in the dorsal and ventral premotor cortex. J Neurophysiol 102:3280-3294. 